\title{
Clinical and Laboratory Profile of Children with Dengue Fever and Predictors of Severe Dengue
}

\author{
Sahana $\mathbf{M}^{1}$, Devakumar VK ${ }^{1}$, Santhosh Kumar $A^{1}$ \\ ${ }^{1}$ Department of Pediatrics, Government Medical College, Thiruvananthapuram, Kerala. \\ Corresponding Author: Devakumar V K
}

\begin{abstract}
Objectives: To study the Clinical and Laboratory profile of children upto 12 years of age with Dengue fever and to study the predictors of Severe Dengue among these children.

Methods:

Design: Descriptive study over 1 year 9 months. Setting: SAT hospital, Thiruvananthapuram, a tertiary care center.

Study Population: 204 children aged 1month to 12 years admitted with serologically confirmed dengue fever without any co-infections; consecutive sampling.

Procedure: Relevant history, detailed physical examination and investigations were noted. Patients were classified for their severity based on DENGUE guidelines-WHO 2009. Children were followed up till discharge during hospital stay. Categorical values were expressed as proportions and quantitative variables in mean and standard deviation. Association between various study variables and severe dengue were statistically analysed.
\end{abstract}

Results: Mild dengue was seen in 96 children $(47.1 \%)$, moderate dengue in 78 children $(38.2 \%)$ and severe dengue in 30 children $(14.7 \%)$. Mean age of presentation was 6.4 years. Age distribution- infants were $4.4 \%$, $38.7 \%$ were between 1 year and 5 years; $56.9 \%$ were between 6 and 12 years. $51 \%$ of children were males.

Common clinical presentation was fever $(100 \%)$, vomiting (46.6\%), abdominal pain $(32.4 \%)$, lethargy $(24.5 \%)$, myalgia(22\%), headache $(16.7 \%)$ and reduced appetite $(15.7 \%)$. Mean fever duration was 5 days. Hypotension was noted in $13.2 \%$, Tender hepatomegaly in $44 \%$, signs of plasma leakage in $6 \%$, hypoalbuminemia in $7.8 \%$. Total number of death was 2(1\%).

Variables with significant association with severe dengue on univariate analysis were Lethargy ( $\mathrm{p}-<0.001$, OR-11.6), reduced appetite (p- <0.001,OR-17.14), mucosal bleed ( $<<0.001$, OR- 15.45), Fever spike $>39^{\circ} \mathrm{C}$ (p- 0.001 , OR4.3 ), tender hepatomegaly (p- 0.001 , OR- 4.2), decreased urine output ( $\mathrm{p}<0.001$, OR- 23.04), hypoalbuminemia ( $\mathrm{p}<0.001$, OR-43.5), platelet count below 80,000 ( $\mathrm{p}<0.001$, OR-4.43), SGOT above 85 ( $\mathrm{p}<0.001$, OR-4.9).

According to the maximum area under ROC curve, a cutoff of platelet count 80,000 had $75 \%$ sensitivity and $60 \%$ specificity for predicting severe dengue.

Conclusion: Proportion of children developed severe dengue was $14.7 \%$. Independent predictors of severe dengue after multiple logistic regression were Lethargy, Reduced appetite, Reduced urine output and Hypoalbuminemia.

Key Words: severe dengue; urine output; shock; hypoalbuminemia.

\section{INTRODUCTION}

Dengue is the most important rapidly emerging mosquito borne disease in the world. Therising burden of dengue has been a critical threat to population health. 50-100 million dengue infections occur every year as estimated by WHO. $75 \%$ of the global population exposed to dengue are in South East Asia Region and Western Pacific region $^{[1]}$.

Attack rate among susceptibles is approximately 40-50 \% during epidemics; every year, around 5,00,000 patients with 
severe dengue require hospitalization of which $2.5 \%$ patients succumb to death. Case fatality rate of severe dengue is as high as $20-25 \%$ without appropriate treatment ${ }^{[1]}$.

Environmental changes, spread of vectors to new areas, raising trend of international travel and trade, rural to urban migration have led to the changes in the trend of dengue fever. Main reasons for difficulty in controlling dengue are non availability of specific treatment, lack of effective vaccine as well as difficulties in controlling vector.

Mortality from dengue can be reduced by timely implementation of appropriate clinical management which includes early clinical and laboratory diagnosis, judicious intravenous rehydration, training health care personnel, timely and prompt referral from primary health-care centers. Integrated vector management- effective urban and household water management, implementing better outbreak prediction through coordinated epidemiological surveillance are also equally important in reducing morbidity.

The spectrum of dengue viral infection is wide, ranging from undifferentiated fever to dengue hemorrhagic fever and dengue shock syndrome. Prior to the critical phase, it is difficult to differentiate between mild and severe dengue. During the early stages of dengue, presence of nonspecific febrile illness makes the diagnosis strikingly difficult which leads to inefficient treatment resulting in rise in morbidity and mortality. If not promptly treated, severe dengue may lead to rapid death especially in pediatric age group. The present study was conducted to explore a set of parameters, preferably routine that could be used as the indicators for severity of infection. Only a small subset of dengue infection will progress to severe dengue. The focus of the study is to identify the children at high risk of progression to severe dengue who can be benefitted from close observation and early intervention.

\section{METHODS}

This descriptive study was conducted in SAT Hospital, Thiruvananthapuram, Kerala, a tertiary care center over a period of 1 year 9 months (December 2017 to September 2019). 204 children aged 1month to 12 years admitted with serologically confirmed dengue fever without any co-infections by consecutive sampling were enrolled in the study.

Relevant history from primary care taker \& detailed physical examination was done at the time of admission. The study variables include demographic variables, Nutritional status, presence of warning signs as per National dengue guidelines 2014, duration of fever, Fever with temperature more than $39{ }^{\circ} \mathrm{C}$ (measured at axilla), hypotension, urine output, hepatomegaly, duration of hospital stay, blood investigations - hemoglobin, total count, platelet count, packed cell volume, serum transaminases, serum albumin, Serum sample for Dengue NS1 / Ig M ELISA depending on day of illness. Children were classified for their severity based on DENGUE guidelines-WHO 2009. Children were followed up till discharge during hospital stay.

Categorical values were expressed as proportions and quantitative variables in mean and standard deviation. Association between various study variables and severe dengue were statistically analysed. Categorical values were expressed as proportions; Chi-square test was used for categorical values. Quantitative variables were expressed as mean and standard deviation. ' $t$ ' test was used for comparison of mean. Odds ratio, confidence interval and significance was noted to study the association. Appropriate statistical tests of significance were applied and factors associated with severe dengue were explored.

\section{OPERATIONAL DEFINITION: \\ Clinical criteria of dengue fever:}

An acute febrile illness of 2- 7 days duration with two or more of the following 
manifestations: headache, retro-orbital pain, myalgia, arthralgia, rash, hemorrhagic manifestations. Severe dengue is defined as at least one of the following (according to DENGUE-guidelines for diagnosis, treatment, prevention \& control- WHO 2009)

1) severe plasma leakage leading to i) shock (circulatory failure manifested by rapid, weak pulse \& narrow pulse pressure $</=20 \mathrm{~mm} \mathrm{Hg}$ or hypotension for age, cold clammy skin) ii)fluid accumulation with respiratory distress

2) dengue fever with severe bleeding

3) severe organ involvement (i) CNSImpaired consciousness, encephalopathy, encephalitis, intracranial bleed. (ii) GIT-Acute hepatitis/ fulminant hepatitis with liver enzymes SGOT/PT >1000U/L, hepatic failure, pancreatitis.(iii)CVS- Cardiac arrhythmia, myocarditis, pericardial effusion ( iv) Renal- Acute renal failure, hemolytic uremic syndrome, acute tubular necrosis (v) RespiratoryPulmonary edema, ARDS, pulmonary hemorrhage (vi) Eye- macular hemorrhage, visual impairment, optic neuritis.

- Urine output of less than $0.5 \mathrm{ml} / \mathrm{kg}$./hour for at least 6 hours was taken as reduced urineoutput.

- Liver of at least $2 \mathrm{~cm}$ palpable below right costal margin at mid clavicular line was takenas hepatomegaly.

\section{RESULTS}

A total of 204 children met the inclusion criteria and were enrolled in the study. The study population were classified in to three age groups; 9 children were below 1 year; 79 children were between 1 year and 5 years; 116 children were between 5 years 1 month and 12 years. Mean age of presentation is 6.4 years. $51 \%$ of the study population were males. Common clinical presentation was fever (100\%), vomiting (46.6\%), abdominal pain (32.4\%), lethargy $(24.5 \%)$, myalgia (22\%), headache $(16.7 \%)$, reduced appetite $(15.7 \%)$, rash $(12.7 \%)$, mucosal bleed $(5.9 \%)$. Mean duration of fever was 5 days. 82 children had fever spike with temperature above $39{ }^{\circ} \mathrm{C}$. Hypotension was documented in 27 children (13.2\%). 37 children (18.1\%) had decreased urine output on the day of admission. Altered sensorium was present in 3\% of children. Hypoalbuminemia was noted in 16 children.40 children have received IV fluid during hospital stay.152 children $(74.5 \%)$ have reached health care facility within 5 days of onset of illness. Dengue NS1 was positive in $35.8 \%$ of children; Dengue Ig M was positive in $57.8 \%$ and both were positive in $6.4 \%$.

Mild dengue was seen in 96 children (47.1\%), moderate dengue in 78 children $(38.2 \%)$ and severe dengue in 30 children (14.7\%). 104 children (51\%) presented with warning signs. The percentage of severe dengue in study population is $14.7 \%$. Average days of Hospital stay was 4.4 days. Two children were expired in the study population. Mortality rate was $1 \%$.

Laboratory profile of the study population: mean $\mathrm{Hb}-11.82$; mean platelet count-1.2 lakh; mean PCV-35.2; mean SGOT/SGPT-140/80. Mann- Whitney U test was done to compare the mean of Hemoglobin, Total count, Platelet count, Packed cell volume, SGOT and SGPT between severe dengue and non severe dengue. Statistically significant association was found with thrombocytopenia and elevated SGOT and SGPT.

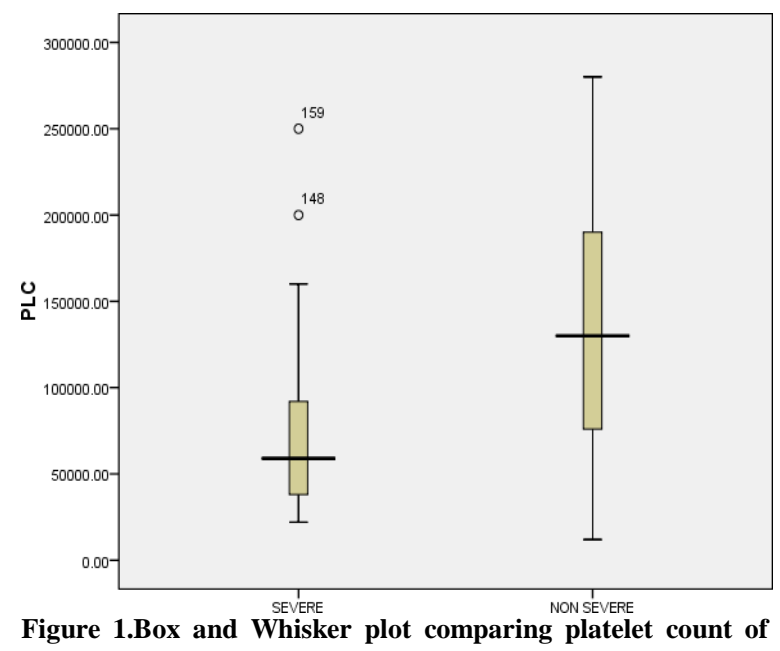
severe and non severe dengue. 
A single platelet count cutoff best associated with severe dengue was identified according to the maximum area under the receiver operating characteristic curve. A cutoff of platelet count 80,000 had $75 \%$ sensitivity and $60 \%$ specificity for predicting severe dengue (figure 2). A single SGOT cutoff best associated with severe dengue was identified according to the maximum area under the receiver operating characteristic curve. A cutoff of SGOT 85 had $70 \%$ sensitivity and $68 \%$ specificity for predicting severe dengue (figure 3 ).

$$
\text { Variables with significant }
$$

association with severe dengue on univariate analysis (table1) were Lethargy (p-<0.001, OR-11.6), reduced appetite (p$<0.001$,OR- 17.14), mucosal bleed $\left(\mathrm{p}<0.001\right.$, OR- 15.45), Fever spike $>39{ }^{\circ} \mathrm{C}$ (p- 0.001, OR- 4.3), tender hepatomegaly (p- 0.001, OR- 4.2), decreased urine output (p <0.001, OR- 23.04), hypoalbuminemia ( $\mathrm{p}$ $<0.001$, OR-43.5), platelet count below 80,000 (p <0.001, OR-4.43), SGOT above 85 ( $\mathrm{p}<0.001$, OR-4.9). The variables with $\mathrm{p}$ value less than 0.05 were included in multivariate logistic regression analysis. Independent predictors of severe dengue after Multiple Logistic Regression were Lethargy, Reduced Appetite, Reduced Urine Output and Hypoalbuminemia (table 2)

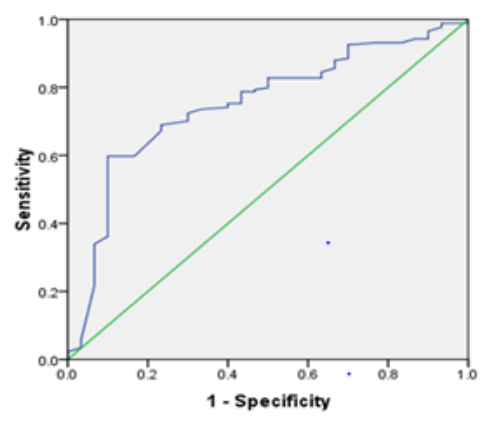

Area Under The Curve-Test Result Variable: Platelet Count

\begin{tabular}{|l|l|l|l|c|}
\hline Area & \multirow{2}{*}{ Std Error } & \multirow{2}{*}{ p value } & \multicolumn{2}{|c|}{$95 \%$ confidence Interval } \\
\cline { 4 - 5 } & & & Lower & Upper \\
\hline 0.75 & $\mathbf{0 . 0 4 7}$ & $<\mathbf{0 . 0 0 1}$ & $\mathbf{0 . 6 5}$ & $\mathbf{0 . 8 4}$ \\
\hline
\end{tabular}

Figure 2.Receiver Operating Characteristic Curve ( ROC CURVE) - Test Result Variable:Platelet Count

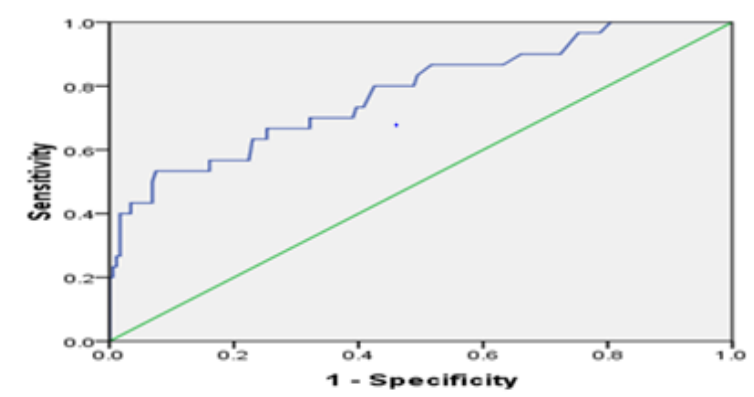

Area Under The Curve-Test Result Variable: SGOT

\begin{tabular}{|l|l|l|l|c|}
\hline Area & Std & p value & \multicolumn{2}{|c|}{$95 \%$ confidence Interval } \\
\cline { 4 - 5 } & Error & & Lower & Upper \\
\hline 0.778 & $\mathbf{0 . 0 4}$ & $<\mathbf{0 . 0 0 1}$ & $\mathbf{0 . 6 8}$ & $\mathbf{0 . 8 7}$ \\
\hline
\end{tabular}

Figure 3 Receiver Operating Characteristic Curve - Test Result Variable: SGOT

Table 1 variables found to be significant in univariate analysis.

\begin{tabular}{|l|l|l|l|l|l|l|l|}
\hline & & $\begin{array}{l}\text { Severe dengue } \\
\mathbf{n = 3 0}\end{array}$ & $\begin{array}{l}\text { Non severe dengue } \\
\mathbf{n = 1 7 4}\end{array}$ & p value & OR & $\begin{array}{l}\text { 95\% CI } \\
\text { lower }\end{array}$ & $\begin{array}{l}\text { 95\% CI } \\
\text { upper }\end{array}$ \\
\hline 1. & Lethargy & 21 & 29 & $<0.001$ & 11.66 & 4.855 & 28.03 \\
\hline 2. & Reduced appetite & 18 & 14 & $<0.001$ & 17.143 & 6.886 & 42.67 \\
\hline 3. & Mucosal bleed & 8 & 4 & $<0.001$ & 15.455 & 4.298 & 55.57 \\
\hline 4. & Fever $>39$ C & 21 & 9 & 0.001 & 4.308 & 1.863 & 9.959 \\
\hline 5. & Tender hepatomegaly & 14 & 30 & 0.001 & 4.2 & 1.853 & 9.518 \\
\hline 6. & Reduced urine output & 21 & 16 & $<0.001$ & 23.042 & 9.046 & 58.69 \\
\hline 7. & Hypoalbuminemia & 13 & 3 & $<0.001$ & 43.588 & 11.2 & 168.258 \\
\hline 8. & Platelet count below80000 & 18 & 44 & $<0.001$ & 4.43 & 1.97 & 9.92 \\
\hline 9. & SGOT above 85 & 21 & 56 & $<0.001$ & 4.917 & 2.11 & 11.42 \\
\hline
\end{tabular}

Table 2 Independent predictors of Severe dengue ( Multiple Logistic Regression)

\begin{tabular}{|l|l|l|l|l|l|l|l|l|}
\hline & & $\begin{array}{l}\text { Severe dengue } \\
\mathbf{n = 3 0}\end{array}$ & $\begin{array}{l}\text { Non severe dengue } \\
\mathbf{n = 1 7 4}\end{array}$ & $\mathbf{d f}$ & $\mathbf{p}$ value & OR & $\begin{array}{l}\mathbf{9 5 \%} \text { CI } \\
\text { lower }\end{array}$ & $\begin{array}{l}\text { 95\% CI } \\
\text { upper }\end{array}$ \\
\hline 1. & Lethargy & 21 & 29 & 1 & 0.002 & 7.8 & 2.08 & 29.3 \\
\hline 2. & Reduced appetite & 18 & 14 & 1 & 0.024 & 5.09 & 1.24 & 20.9 \\
\hline 3. & Reduced urine output & 21 & 16 & 1 & 0.001 & 9.04 & 2.61 & 31.37 \\
\hline 4. & Hypoalbuminemia & 13 & 3 & 1 & 0.009 & 16.34 & 2.01 & 132.8 \\
\hline
\end{tabular}

\section{DISCUSSION}

Out of total 204 children, mild dengue cases were $96(47.1 \%)$, moderate dengue cases were $78(38.2 \%)$ and severe dengue cases were $30 \quad(14.7 \%)$. Age distribution- infants were $4.4 \%$,

$38.7 \%$ were between 1 year and 5 years; $56.9 \%$ were between 6 and 12 years. 
More prevalence of dengue in 6-12years age group is probably due to more involvement in outdoor activities during the day time exposing them to a higher risk of mosquito bites. According to guidelines by WHO ${ }^{[1]}$, infancy was considered as a risk group. Several studies showed that young and old ages were associated with severe dengue ${ }^{[2,3]}$. But Pongpon et al found that age more 6 years as a risk factor for DHF ${ }^{[4]}$. Also Yolando et al reported age more than 5 years to be a risk factor forsevere dengue ${ }^{[5]}$. In our study, age below 5 years was not found to be associated with severe dengue. This might be due to lower proportion of infants in our study population $(4.4 \%)$. In our study, $51.5 \%$ of study population (105 children) were males and rest $48.5 \%$ were females (99 children). Male female ratio was 1.06:1. No significant association was found between gender and severe dengue in our study. Jiu L et al ${ }^{[6]}$, Pongpon et al ${ }^{[4]}$ and Maron et al ${ }^{[7]}$ in their study also reported the same. Male preponderance was seen in our study probably due to more involvement of boys in outdoor activities with increased risk of exposure to mosquitoes.

In our study, 135 children $(66.5 \%)$ had no protein energy malnutrition; Grade 1 PEM was seen in 44 children (21.6\%), Grade 2 PEM in 16 children (7.8\%), Grade 3 PEM in 4 children (2\%), Obesity was seen in 5 children (2.5). A study on 4,532 children confirmed of dengue infection compared to control showed that children with under nutrition had a greater risk of DSS ${ }^{[8]}$. In our study, PEM was not found to be associated with severe dengue. In another study by Maron et al, nutrition does not appear to be a risk factor for severe forms of dengue infection, nor does malnutrition appear to be predictive of good outcomes ${ }^{[7]}$. Nutritional status is easy to assess, but further studies are needed before we can use it as a predictor for severe dengue.

Lethargy, Reduced appetite, Mucosal bleed, Fever spike with temperature $>39 \circ \mathrm{C}$ - these variables were found to have statistically significant association with severe dengue on univariate analysis. Vomiting was considered as one of the feature of severe dengue but we did not find the same in our study. Abdominal pain was reported as another prognostic factor for severe dengue $[9,10]$. It could be caused by gastrointestinal bleeding and/or hepatomegaly. Junia et al in their study proposed that during the shock or pre-shock state, blood supply to visceral organs was reduced causing tissue hypoxia followed by abdominal pain ${ }^{[9]}$. Bleeding manifestations, hepatomegaly have been found to be predictive of severity in some studies ${ }^{[4,10]}$.

21 out of 30 severe dengue cases $(70 \%)$ presented with fever spike of temperature above $39 \circ \mathrm{C}$. There was statistically significant association between fever spike with temperature above $39^{\circ} \mathrm{C}$ and severe dengue as also reported by Alvarado-castro et al ${ }^{[11]}$.

There is loss of plasma volume as result of capillary leak in patients of DHF. Those with pleural effusion, ascites, gall bladder edema suggesting capillary leak were found to be significantly associated with severe dengue in our study. This was also reported by Chacko et al ${ }^{[12]}$. Mild hemorrhagic manifestations like petechiae and mucosal membrane bleeding are commonly seen in DHF [13,14]. Severe bleeding/internal hemorrhage consists of melena, haematemesis, hematuria and/or menorrhagia ${ }^{[13]}$. Melena was seen in $1.5 \%$ of our children.

Melena constituted the most common form of internal bleed by Shah et al. ${ }^{[3]}$. Haematemesis was reported as the most common manifestation in the studies by Narayanan et al. ${ }^{[15]}$; Hammond et al ${ }^{[2]}$ reported that frequency of internal hemorrhage increased with increase in age of the patients. Mucosal bleeding was significantly associated with severe dengue in our study as reported by other studies ${ }^{\text {[12] }}$. An erythematous or macular papular rash may appear in the acute febrile phase along with other constitutional symptoms ${ }^{[9]}$. When the presence of rash was analyzed 
with reference to severe dengue, no significance could be found in our study. Tender hepatomegaly was seen in $46.6 \%$ of severe dengue cases and $17 \%$ of non severe dengue cases. There was statistically significant association between tender hepatomegaly and severe dengue $(\mathrm{p}=0.001)$. This was also reported by several studies $[4,5]$

In our study, lethargy was significantly associated with severe dengue in both univariate and multivariate analysis. This was also noticed by other studies ${ }^{[16]}$. 27 children were found tohave hypotension $(13.2 \%)$. Decreased urine output was seen in $70 \%$ of severe dengue cases and $22 \%$ of non severe dengue cases. There was statistically significant association between decreased urine output and severe dengue.

Among the lab parameters, statistically significant association was found between low platelet count and elevated transaminases level. Thrombocytopenia was reported as a risk factor by Yolando et al ${ }^{[5]}$. Significant association of platelet count $<10000$, $<50000$ and $\leq 75000 \mathrm{~mm}^{3}$ has been reported with occurrence of DSS by Chacko et al., Hammond et al. and Pham et al., respectively $[2,10,12]$. The earliest hematological abnormality is a progressive decline in total WBC count in patients of dengue. A leucopenia of $\mathrm{WBC}<5000 \mathrm{~mm}^{-3}$ has been suggested by Kalayanarooj et al. ${ }^{[14]}$ to predict the onset of DHF. The WBC count $<4000 \mathrm{~mm}^{-3}$ was significantly associated with DSS in this study as reported by Chacko et al. ${ }^{[12]}$.

Though there is usual rise in the serum liver enzymes ${ }^{[10]}$ with dengue infection, but whether they predict the risk of DSS in patients with DHF could not be stated with certainty as many studies refute and support the same. Also, what levels of SGPT or SGOT predict the worst outcome is also conflicting as Parkash et al. ${ }^{[16]}$ have taken SGPT level of 300 as a measure of worst outcome in dengue patients, whereas latest WHO guidelines (13) have taken
SGPT/SGOT value $\geq 1000$ as one of the feature of severe dengue.

Hypoalbuminemia was present in $43.3 \%$ of severe dengue cases and was found to have significant association with severe dengue $(\mathrm{p}<0.001)$ as reported by Halstead et al., ${ }^{[17]}$.

\section{CONCLUSION}

Proportion of children developed severe dengue was $14.7 \%$. Independent predictors of severe dengue after multiple logistic regression were Lethargy, Reduced appetite, Reduced urine output and Hypoalbuminemia.

Contributors: Sahana M: designed the methodology, collected and analysed data and prepared the manuscript; Devakumar VK: conceived the idea, guided conduct of the study, critically reviewed the manuscript; Santhosh Kumar A: interpreted the results, critically reviewed the manuscript. All authors approved the final version of manuscript and are accountable for all aspects related to the study.

\section{Acknowledgement: None}

Conflict of Interest: None

\section{Source of Funding: None}

\section{Ethical Approval: Approved}

\section{REFERENCES}

1. Dengue-National-Guidelines-2014

Compressed.pdf [Internet]. [cited 2019 Oct 6]. Available from: http://pbhealth.gov.in/Dengue-NationalGuidelines-2014\%20Compressed.pdf

2. Hammond SN, Balmaseda A, Pérez L, Tellez Y, Saborío SI, Mercado JC, et al. Differences in dengue severity in infants, children, and adults in a 3-year hospitalbased study in Nicaragua. Am J Trop Med Hyg. 2005 Dec;73(6):1063-70.

3. Shah I, Deshpande GC, Tardeja PN. Outbreak of dengue in Mumbai and predictive markers for dengue shock 
syndrome. J Trop Pediatr. 2004;50(5):3015.

4. Prognostic Indicators for Dengue Infection Severity | Pongpan | International Journal of Clinical Pediatrics [Internet]. [cited 2019 Oct 12]. Available from: https://theijcp.org/index.php/ijcp/article/vie $\mathrm{w} / 73 / 68$

5. Yolanda N, Alfan H. Initial clinical and laboratory profiles to predict pediatric dengue infection severity. Paediatr Indones [Internet]. $2017 \quad$ [cited 2019 Oct 15];57(6):303-9. Available from: https://paediatricaindonesiana.org/index.php /paediatrica- indonesiana/article/view/1628

6. Liu J, Tian X, Deng Y, Du Z, Liang T, Hao Y, et al. Risk Factors Associated with Dengue Virus Infection in Guangdong Province: A Community-Based CaseControl Study. Int J Environ Res Public Health [Internet]. 2019 Feb [cited 2019 Oct 15];16(4). Available from: https://www.ncbi.nlm.nih.gov/pmc/articles/ PMC6406885/

7. Marón GM, Clará AW, Diddle JW, Pleités EB, Miller L, MacDonald G, et al. Association between Nutritional Status and Severity of Dengue Infection in Children in El Salvador. AmJ Trop Med Hyg [Internet]. 2010 Feb [cited 2019 Oct 12];82(2):324-9. Available from: https://www.ncbi.nlm.nih.gov/pmc/articles/ PMC2813176/

8. Kalayanarooj S, Nimmannitya S. Is dengue severity related to nutritional status? Southeast Asian J Trop Med Public Health. 2005 Mar;36(2):378-84.

9. Junia J, Garna H, Setiabudi D. Clinical risk factors for dengue shock syndrome in children. Paediatr Indones [Internet]. 2007 Feb 28 [cited 2019 Oct 12];47(1):7-11. Available from: https://paediatricaindonesiana.org/index.php /paediatrica-indonesiana/article/view/257

10. Pham TB, Nguyen TH, Vu TQH, Nguyen TL, Malvy D. [Predictive factors of dengue shocksyndrome at the children Hospital No. 1, Ho-chi-Minh City, Vietnam]. Bull Soc Pathol Exot1990. $2007 \mathrm{Feb}$;00(1):43-7.

11. Alvarado-Castro VM, Ramírez-Hernández E, Paredes-Solís S, Legorreta-Soberanis J,
Saldaña-Herrera VG, Salas-Franco LS, et al. Clinical profile of dengue and predictive severity variables among children at a secondary care hospital of Chilpancingo, Guerrero, Mexico: case series. Bol Méd Hosp Infant México Engl Ed [Internet]. 2016 Jul 1 [cited 2019 Oct 16];73(4):23742. Available from: http://www.sciencedirect.com/science/articl e/pii/S2444340917000218

12. Chacko B, Subramanian G. Clinical, laboratory and radiological parameters in children with dengue fever and predictive factors for dengue shock syndrome. J Trop Pediatr. 2008 Apr;54(2):137-40.

13. Balmaseda A, Hammond SN, Pérez MA, Cuadra R, Solano S, Rocha J, et al. Short report: assessment of the World Health Organization scheme for classification of dengue severity in Nicaragua. Am J Trop Med Hyg. 2005 Dec;73(6):1059-62.

14. Kalayanarooj S, Vaughn DW, Nimmannitya S, Green S, Suntayakorn S, Kunentrasai N, etal. Early clinical and laboratory indicators of acute dengue illness. J Infect Dis. 1997 Aug;176(2):313-21.

15. Narayanan M, Aravind MA, Thilothammal N, Prema R, Sargunam CSR, Ramamurty N. Dengue fever epidemic in Chennai--a study of clinical profile and outcome. Indian Pediatr.2002 Nov;39(11):1027-33.

16. Almas A, Parkash O, Akhter J. Clinical factors associated with mortality in dengue infection at a tertiary care center. Southeast Asian J Trop Med Public Health. 2010 Mar;41(2):333-40.

17. Halstead SB, Halstead SB. Predictors of dengue severity. J Pediatr (Rio J) [Internet]. 2016Oct [cited 2019 Oct 16];92(5):429-31. Available from: http://www.scielo.br/scielo.php?script=sci_a bstract\&pid=S0021-

$75572016000600429 \& \operatorname{lng}=$ en\&nrm=iso\&tl $\mathrm{ng}=\mathrm{en}$

How to cite this article: Sahana M, Devakumar VK, Santhosh Kumar A. Clinical and laboratory profile of children with dengue fever and predictors of severe dengue. International Journal of Research and Review. 2021; 8(8): 17. DOI: https://doi.org/10.52403/ijrr.20210801 